\section{Percepción de las relaciones familiares y nivel de resiliencia en el adulto mayor: un estudio descriptivo-comparativo}

\section{Perception of family relations and resilience level in the older adults: A descriptive-comparative study}

\author{
Alejandro Morales-Jinez',** \\ Elizabeth Fajardo-Ramos ${ }^{2}$ \\ Francisco Javier López-Rincón ${ }^{3}$ \\ Alicia Ugarte-Esquivel ${ }^{4}$ \\ Yolanda Ivonne Trujillo-León ${ }^{5}$ \\ Luz Elena Rodríguez-Mejía ${ }^{5}$
}

1. Doctor en Ciencias de Enfermería. Universidad Autónoma de Coahuila. Escuela de Licenciatura en Enfermería. Unidad Torreón. México.

2. Magíster en Enfermería. Universidad del Tolima. Facultad de Ciencias de la Salud. Ibagué. Colombia.

3. Doctor en Educación. Universidad Autónoma de Coahuila. Escuela de Licenciatura en Enfermería. Unidad Torreón. México.

4. Maestra en Enfermería con énfasis en salud comunitaria. Universidad Autónoma de Coahuila. Escuela de Licenciatura en Enfermería. Unidad Torreón. México.

5. Maestra en Enfermería. Universidad Autónoma de Coahuila. Escuela de Licenciatura en Enfermería. Unidad Torreón. México.

${ }^{*}$ Autor para correspondencia.

Correo electrónico: alejandro_morales@uadec.edu.mx (Alejandro Morales-Jinez).

\section{RESUMEN}

Objetivo: Comparar la percepción de relaciones familiares y la resiliencia en adultos mayores. Metodología: Estudio cuantitativo, descriptivo, comparativo, prospectivo y de corte transversal; la muestra estuvo conformada por 130 adultos mayores de 60 años de diversas comunidades de la Región Lagunera, al norte de México, seleccionados por un muestreo no probabilístico a conveniencia. Para la recogida de datos se utilizaron el Test de percepción de relaciones familiares del anciano y la Escala de Resiliencia de Connor-Davidson. Los datos se analizaron en el programa SPSS v20 y se aplicaron frecuencias para variables cualitativas, medidas de tendencia central y dispersión para variables cuantitativas, y para la comparación de los grupos se utilizó la prueba de Kruskal Wallis. Resultados: La mayor parte de la muestra eran mujeres $(76,9 \%)$ y el $56,2 \%$ no tenía pareja. Los tres grupos no mostraron diferencia significativa en las características sociodemográficas. El análisis inferencial mostró diferencia significativa entre la percepción de la relación familiar y el nivel de resiliencia en los adultos mayores, en los grupos de percepción disarmónica con los grupos de percepción poco armónica y armónica. Conclusiones: Existe diferencia entre la percepción de la relación familiar y el nivel de resiliencia en el adulto mayor; se observa que a mejor percepción de la relación familiar por el adulto más se incrementa el nivel de resiliencia.

PALABRAS CLAVE: Resiliencia psicológica, relaciones familiares, adulto mayor.

\section{ABSTRACT}

Objective: To compare the perception of family relations and resilience in older adults. Methodology: Quantitative, descriptive, comparative, prospective and cross-sectional study; the sample consisted of 130 adults over 60 years of age from diverse communities of the Lagunera

Region, in northern Mexico, selected by a non-probabilistic sample at convenience. For the collection of data, the Elderly Family Relationship Perception Test and the Connor-Davidson Resilience Scale were used. The data were analyzed in the SPSS v20 program and frequencies were applied for qualitative variables, measures of central tendency and dispersion for quantitative variables, for comparison of the groups used the Kruskal Wallis test. Results: The majority of the sample were women (76.9\%) and 56.2\% did not have a partner. The three groups showed no significant difference in sociodemographic characteristics. The inferential analysis shows a significant difference between the perception of the family relationship and the level of resilience in the older adults, in the groups of disharmonic perception with the groups of perception that are not harmonious and harmonious. Conclusions: There is a difference between perception of the family relationship and the level of resilience in the older adult, it is observed the better perception of the family relationship by the older adult increases the level of resilience.

KEYWORDS: Psychological resilience, family relations, aged.

\section{- INTRODUCCIÓN}

El mundo atraviesa por una notable transición demográfica caracterizada por el incremento de personas mayores de 60 años. Estos cambios en la población se han producido de forma gradual en países desarrollados; sin embargo, en los países considerados en desarrollo, como es el caso de México, se ha dado de forma súbita y es el resultado de la disminución en la natalidad y la mortalidad, así como de los avances de investigación tecnológica y de cuidados a la salud ${ }^{1,2}$. Aunado al cambio demográfico, se ha dado paso a una transición epidemiológica; por lo tanto, las necesidades de salud han cambiado, ya que existe mayor frecuencia de enfermedades crónico-degenerativas como resultado de los estilos de vida no saludables, el estrés, el sobrepeso, la obesidad, y el consumo excesivo de tabaco y alcohol, entre otras causas 2 .

A pesar de que el adulto mayor es considerado en el ámbito de la salud como un grupo vulnerable por las condiciones poco favorables en el 
entorno donde se desarrolla, como son: maltrato, violencia, problemas familiares, bajos o nulos ingresos económicos y detrimento del estado de salud $^{3}$, limitación del acceso a los servicios de salud, predominio del género femenino ${ }^{4}$, por mencionar algunos, se observa que existen personas mayores con un alto nivel de funcionalidad física y mental, a pesar de las circunstancias hostiles en las que viven y se desenvuelven ${ }^{3}$. En otras palabras, los adultos mayores son resilientes.

La resiliencia es la capacidad que tienen las personas para afrontar o recuperarse de forma exitosa cuando se presentan situaciones adversas en la vida, manteniendo estables los niveles de la salud a nivel físico y psicológico ${ }^{3-7}$. Recientemente, se ha desarrollado el concepto de resiliencia aplicado al ciclo de vida de la vejez y se define como un proceso multidimensional de adaptación positiva ante las adversidades, aunque se encuentren presentes algunas limitaciones y dificultades propias del envejecimiento $^{3-9}$.

Los resultados de los estudios de investigación muestran que los adultos mayores resilientes manifiestan efectos positivos en la salud, ya que a mayor nivel de resiliencia existe menor nivel de depresión, mejor percepción en el nivel de salud, fortalecimiento del envejecimiento exitoso, aumento de la longevidad ${ }^{10}$, menor precepción en el nivel de dolor crónico, mejor nivel de salud mental y física ${ }^{11}$, disminución de los efectos negativos del cortisol ${ }^{12}$ y por lo tanto, incremento en la calidad de vida de la persona mayor ${ }^{13}$.

Existen algunos factores que ayudan a desarrollar y mantener altos niveles de resiliencia en el adulto mayor. Entre estos factores se encuentran: la espiritualidad, el apego religioso, las experiencias personales previas (positivas o negativas), los factores contextuales-culturales y la familia 5 . El apoyo social percibido por el adulto mayor, es decir, la ayuda de la pareja, redes familiares y/o amistades cercanas, se asocia con el desarrollo de la resiliencia, ya que permiten perfeccionar los estilo de afrontamiento a las adversidades, promueven el optimismo, la esperanza y emociones positivas ${ }^{10}$; sin embargo, el tipo de relaciones familiares percibidas por el adulto mayor es un tema en el que no se ha profundizado y surge el siguiente interrogante: ¿existe diferencia en el nivel de resiliencia de los adultos mayores y el tipo de relación familiar percibida? El objetivo general de este estudio fue, por tanto, comparar la percepción de relaciones familiares y la resiliencia en adultos mayores de 60 años de las comunidades de la Región Lagunera, al norte de México. Los objetivos específicos fueron: a) identificar el nivel de resiliencia de los adultos mayores y $b$ ) describir la percepción de las relaciones familiares de los adultos mayores.

\section{- METODOLOGÍA}

El presente estudio se realizó bajo el paradigma cuantitativo, con un diseño descriptivo, comparativo, prospectivo y de corte transversal. La población del estudio estuvo formada por los adultos mayores de la zona metropolitana de la Laguna, al norte de México. La muestra fue de 130 adultos mayores, la cual se calculó con el programa estadístico Epidat v4.1 con los siguientes parámetros: un nivel de confianza del 95\% y una potencia del $80 \%$. El muestreo fue no probabilístico a conveniencia y los participantes fueron seleccionados con base en los siguientes criterios: adultos mayores de 60 ańos o más, orientados en tiempo y espacio, que participaran de forma voluntaria. Se excluyó a aquellos adultos mayores solitarios, ya que no tenían familia o no habían tenido ningún tipo de relación con su familia en el último año.

Se aplicó una cédula con datos sociodemográficos que incluía datos como: edad, sexo, estado civil, escolaridad y religión de los participantes. Para obtener los datos sobre la percepción de la relación familiar, se utilizó el Test de percepción de las relaciones familiares en el anciano, que consta de un total de 30 preguntas. Los reactivos miden la percepción de la relación familiar en el adulto mayor de acuerdo con una escala Likert de cinco puntos, donde casi siempre $=5$, muchas veces $=4$, algunas veces $=3$, pocas veces $=2$ y casi nunca $=1 . \mathrm{La}$ escala permite obtener una puntuación máxima de 150 puntos y una mínima de 30. Cuanto mayor es la puntuación, mejor es la relación familiar. La interpretación del instrumento permite crear cuatro categorías de la siguiente forma: de 146 a 150 puntos se perciben relaciones familiares muy armónicas; de 140 a 145 puntos, relaciones familiares armónicas; de 129 a 139 puntos, relaciones familiares poco armónicas, y de 30 a 128 puntos, relaciones familiares disarmónicas. Los autores reportan una confiabilidad de $0,80^{14}$.

Para la medición de la resiliencia se utilizó la Escala de resiliencia de Connor-Davidson (CD-RISC), la cual consta de 25 ítems y emplea una escala de $0-4$, donde 0 = "En absoluto", 1 = "Rara vez", 2 = "A veces", 3 = "A menudo" y 4 = "Casi siempre" y el adulto mayor indica hasta qué punto cada una de las afirmaciones ha sido verdadera en su caso durante el último mes. Las puntuaciones totales oscilan entre 0 y 100; a mayores puntuaciones, mayor resiliencia. La versión original mostró un alfa de Cronbach de 0,89 en población estadounidense y de 0,86 en población española ${ }^{15}$.

Para la recogida de datos se utilizó la técnica de entrevista estructurada, ya que se leía la pregunta del cuestionario, se daban las opciones de respuesta y el entrevistador marcaba la opción que el adulto mayor indicaba; esta se realizó en un espacio tranquilo y libre de distracciones para entablar la conversación. Antes de la recogida de datos se realizó una capacitación a los encuestadores para la cumplimentación de instrumentos, correcta formulación de las preguntas y el registro de las respuestas. El estudio obtuvo la aprobación del comité de ética e investigación de la institución correspondiente con registro 2016/ELEUAC/001. Una vez aprobado el proyecto se procedió a tramitar el permiso con las autoridades correspondientes para aplicar la cédula de datos sociodemográficos y cuestionarios antes especificados. Todos los participantes firmaron el consentimiento informado y se les ofreció un aperitivo al término de la entrevista.

El análisis estadístico de los datos se realizó en el programa SPSS v.20 para Mac. Se aplicó estadística descriptiva como medidas de tendencia central y de dispersión para variables cuantitativas, y frecuencias relativas para variables cualitativas. Para el análisis de confiabilidad de los instrumentos se aplicó el alfa de Cronbach y, finalmente, para comparar los grupos se utilizó el análisis de Kruskal-Wallis para muestras independientes. Se fijó un nivel del 95\% de confianza, considerando significativo cuando $p<0,05$.

\section{- RESULTADOS}

Los adultos mayores de la muestra tenían una edad media de 68,96 $( \pm 6,56)$ años; las edades oscilaron en un rango de 60 a 90 años. La escolaridad medida en ańos de estudio que reportaron los sujetos de la muestra fue en promedio de $6,95( \pm 4,31)$. La escolaridad fue diversa, ya que se encontraron personas mayores que no reportaban estudios, personas mayores con estudios básicos y profesionales. La descripción de otras variables sociodemográficas como el género, estado civil y la religión pueden observarse en la tabla 1 . Al comparar los tres grupos de adultos mayores, categorizados de acuerdo con la percepción de las relaciones familiares, se obtuvieron grupos similares en las características sociodemográficas (tabla 2), ya que los estadísticos aplicados no mostraron diferencias significativas. 
Tabla 1. Características sociodemográficas de la muestra

\begin{tabular}{|l|l|c|c|}
\hline \multicolumn{2}{|c|}{ Característica } & $\begin{array}{c}\text { Frecuencia } \\
\text { absoluta }(\mathbf{n})\end{array}$ & $\begin{array}{c}\text { Frecuencia } \\
\text { relativa (\%) }\end{array}$ \\
\hline \multirow{2}{*}{ Sexo } & Mujer & 100 & 76,9 \\
\hline \multirow{2}{*}{ Estado civil } & Hombre & 30 & 23,1 \\
\hline \multirow{3}{*}{ Religión } & Con pareja & 57 & 43,8 \\
\hline & Sin pareja & 73 & 56,2 \\
\hline & Católica & 101 & 77,7 \\
\hline & Cristiana & 24 & 18,5 \\
\hline & Ninguna & 1 & 0,8 \\
\hline & Otra & 4 & 3,0 \\
\hline
\end{tabular}

Las frecuencias sobre el tipo de relación familiar en los adultos mayores de la muestra en estudio indican que 97 (74,6\%) percibían una relación familiar disarmónica, 26 (20\%) una relación poco armónica y 7 (5,4\%) una relación armónica. En el grupo incluido en este estudio no se encontró ningún participante mayor que percibiera una relación muy armónica.

Al realizar el análisis descriptivo de la resiliencia, se observa una media en la puntuación de 77,99 ( \pm 17,21). El valor mínimo se situó en 8 puntos y el máximo en 100. El primer cuartil (Q1) se encontró en 67 puntos, el segundo cuartil (Q2) en 80 puntos y el tercero (Q3) en 90,25, tal como se observa en la gráfica de cajas (fig. 1).

El análisis de confiabilidad de la Escala de resiliencia de Connor-Davidson y el Test de percepción de las relaciones familiares en el anciano demostraron buena confiabilidad, obteniendo un alfa de Cronbach general de 0,941 y 0,896 , respectivamente.

Finalmente, en el análisis inferencial se muestra una diferencia significativa (Kruskal-Wallis $=14,036, \mathrm{gl}=2, p=0,001$ ) entre el tipo de relación familiar que percibe el adulto mayor y el nivel de resiliencia. Al realizar la comparación por parejas se observa diferencia significativa entre la precepción de relaciones familiares disarmónicas y poco armónicas (Kruskal-Wallis $=-23,566, p=0,014$ ) y entre la precepción de re- laciones familiares disarmónicas y armónicas (Kruskal-Wallis $=-40,841$, $p=0,017)$. Se puede apreciar que a mejor percepción de la relación familiar por el adulto mayor se incrementan las puntuaciones de resiliencia, dando la diferencia significativa entre los adultos mayores que percibieron una relación familiar disarmónica con aquellos que tienen una relación poco armónica y armónica (fig. 2).

\section{DISCUSIÓN}

La muestra estuvo constituida en su mayoría por mujeres adultas mayores, lo cual concuerda con las estadísticas internacionales, en las que se muestra que existe un mayor número de mujeres debido a que la esperanza de vida en el género femenino es superior a la del género masculino, aunque no significa que vivan más años y en mejores condiciones de salud ${ }^{2}$. Asimismo, el nivel educativo de los participantes fue muy diverso, aunque la literatura es muy consistente en que las variables sociodemográficas en adultos mayores y otros grupos de edad no tienen mucha influencia sobre el nivel de resiliencia, ya que el desarrollo del nivel de afrontamiento a la adversidad se debe más a factores de tipo emocional y social ${ }^{16,17}$.

Al realizar el análisis de la relaciones familiares en el adulto mayor, se observa que la mayoría percibe una relación familiar disarmónica, contrario a lo que se reporta en estudios realizados en Cuba, donde la mayoría tiene una percepción de relación familiar armónica y muy armónica $^{18,19}$. La familia es la institución social más cercana e importante para el adulto mayor, ya que representa un apoyo inmediato para la satisfaccción de sus necesidades humanas; sin embargo, las características del contexto social, como son los valores culturales, medios de comunicación, tipo y dinámica de las familias, representan un factor que puede influir en el trato de la familia hacia la persona mayor y, por lo tanto, en la apreciación por parte del adulto mayor y su familia.

El alfa de Cronbach del instrumento CD-RISC para medición de la resiliencia en este estudio fue ligeramente superior al reportado por otros autores en poblaciones de Estados Unidos y España ${ }^{15}$. De igual forma, el alfa de Cronbach para el Test de percepción de las relaciones familiares en el anciano en esta población mostró una

Tabla 2. Comparación de las características sociodemográficas en los grupos de adultos mayores de acuerdo con la percepción de la relación familiar

\begin{tabular}{|c|c|c|c|c|c|}
\hline \multirow{2}{*}{\multicolumn{2}{|c|}{$\begin{array}{l}\text { Característica } \\
\text { sociodemográfica }\end{array}$}} & \multicolumn{3}{|c|}{ Percepción de la relación familiar del adulto mayor } & \multirow[b]{2}{*}{ Análisis estadístico } \\
\hline & & $\begin{array}{l}\text { Relaciones familiares } \\
\text { disarmónicas }\end{array}$ & $\begin{array}{l}\text { Relaciones familiares } \\
\text { poco armónicas }\end{array}$ & $\begin{array}{c}\text { Relaciones familiares } \\
\text { armónicas }\end{array}$ & \\
\hline \multirow{5}{*}{ Edad } & Media & 69,47 & 67,85 & 66,00 & \multirow{5}{*}{ Kruskal-Wallis 2,62; $2 \mathrm{gl} ; p=0,269$} \\
\hline & Mediana & 69,00 & 67,00 & 65,00 & \\
\hline & $\mathrm{DE}$ & 6,70 & 6,41 & 3,92 & \\
\hline & Min-máx & $60-90$ & $60-84$ & $60-73$ & \\
\hline & & $f(\%)$ & $f(\%)$ & $f(\%)$ & \\
\hline \multirow{2}{*}{ Género } & Mujer & $77(59,2)$ & $19(14,6)$ & $4(3,1)$ & \multirow{2}{*}{$\chi^{2}=2,09 ; 2 \mathrm{gl} ; p=0,352$} \\
\hline & Hombre & $20(15,4)$ & $7(5,4)$ & $3(2,3)$ & \\
\hline \multirow{4}{*}{ Religión } & Católica & $77(59,2)$ & $18(13,8)$ & $6(4,6)$ & \multirow{4}{*}{$\chi^{2}=13,76 ; 8 \mathrm{gl} ; p=0,088$} \\
\hline & Cristiana & $19(14,6)$ & $4(3,1)$ & $1(0,8)$ & \\
\hline & Otra & $1(0,8)$ & $3(2,3)$ & $0(0)$ & \\
\hline & Ninguna & $0(0)$ & $1(0,8)$ & $0(0)$ & \\
\hline \multirow[t]{2}{*}{ Estado civil } & Con pareja & $38(29,2)$ & $15(11,5)$ & $4(3,1)$ & \multirow{2}{*}{$\chi^{2}=3,39 ; 2 \mathrm{gl} ; p=0,184$} \\
\hline & Sin pareja & $59(45,4)$ & $11(8,5)$ & $3(2,3)$ & \\
\hline
\end{tabular}




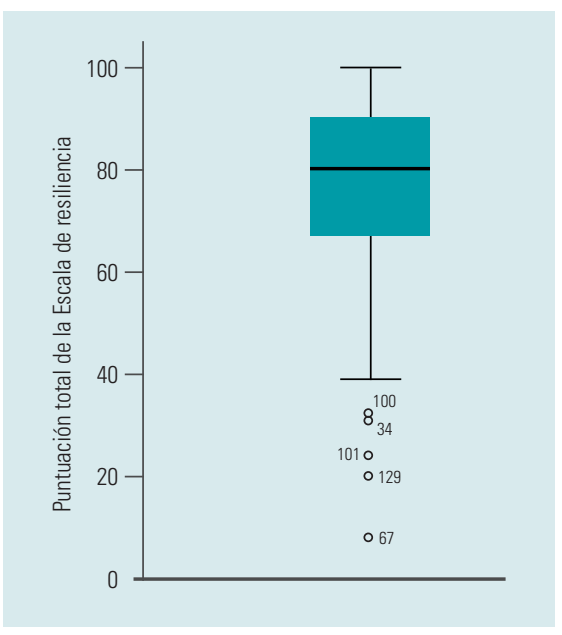

Figura 1. Gráfico de cajas con la representación de la puntuación de la Escala de resiliencia obtenido en la muestra de estudio.

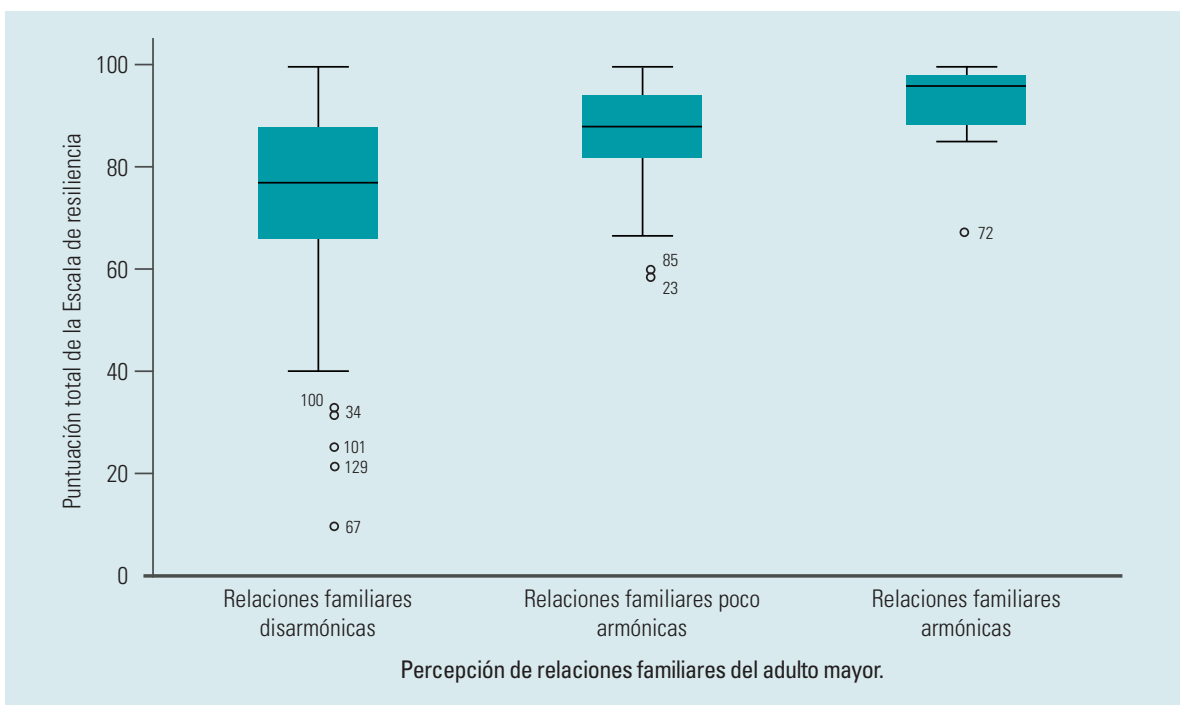

Figura 2. Prueba de Kruskal-Wallis para muestra independientes entre tipo de relación. confiabilidad superior a lo señalado por los autores del instrumento ${ }^{14}$, mostrando que tiene un buen y excelente grado de confiabilidad para la población mexicana.

Al comparar la percepción de las relaciones familiares del adulto mayor con otros autores se muestra que un porcentaje muy bajo tiene una percepción disarmónica de la relación familiar (menos del $5 \%)^{18}$, contrario a lo reportado en este estudio en el que la mayoría (casi el 75\%) percibía una relación familiar disarmónica. Esta diferencia se debe posiblemente a los espacios donde se recogió la información, ya que los estudios fueron realizados en grupos de personas mayores de 60 años, mientras que este estudio se llevó a cabo en el ámbito comunitario, lo que pudo influir de manera importante en la percepción de la relación familiar con la persona mayor.

El promedio de puntuación de resiliencia es ligeramente superior a lo reportado por otros autores; sin embargo, los resultados de esos estudios incluyeron poblaciones con un rango de 18 a 72 años $^{15}$, a diferencia de esta muestra que solo incluyó adultos mayores con un rango de edad entre 60 y 90 ańos, lo cual pudo ser un factor que influyera en el nivel de resiliencia, debido a las experiencias previas con las que contaba este tipo de población.

Finalmente, similar a lo que diversos autores afirman sobre la influencia positiva de la familia o redes familiares en la resiliencia ${ }^{5,10}$, este estudio muestra que la percepción de las relaciones familiares se encuentra asociada al nivel de resiliencia; sin embargo, en la literatura analizada no se encontraron estudios que hablaran sobre la asociación de la percepción de las relaciones familiares con la resiliencia en el adulto mayor.

El alcance de este estudio es limitado, ya que se utilizó un diseño descriptivo y comparativo; se recomienda que para futuros estudios se utilicen diseños con un mayor control de variables externas que pudieran afectar a los resultados de la investigación.

\section{- CONCLUSIONES}

La percepción de las relaciones familiares tiene un papel primordial con el nivel de resiliencia del adulto mayor, lo cual se reflejará a largo plazo en su estado de salud. El promedio del nivel de resiliencia como resultado obtenido indica la influencia de una amplia experiencia positiva y exposición a diversos factores de riesgo y/o protección durante todo el ciclo vital de la persona mayor. Asimismo, se puede seguir fortaleciendo o desarrollando durante la vejez.

Existe diferencia entre el nivel de resiliencia del adulto mayor y la relación familiar percibida; se observa que a mejor percepción de la relación familiar por el adulto mayor se incrementa el nivel de resiliencia. Es necesario diseñar e implementar estrategias de cuidado enfermero con resiliencia, que ayuden a mejorar las relaciones familiares del adulto mayor y su calidad de vida. Mantener el vínculo familiar contribuye al desarrollo de la resiliencia y a una mejor preparación para adaptarse a los cambios que se dan en la vejez

\section{Financiamiento y conflicto de intereses}

El proyecto recibió apoyo financiero del Programa para el Desarrollo Profesional Docente, para el Tipo Superior (PRODEP), México, proceso DSA/103.5/15/7082. Los autores declaran no tener ningún conflicto de intereses.

\section{- BIBLIOGRAFÍA}

1. Helpage International [Internet]. Global AgeWatch. Índice globa de envejecimiento, AgeWatch 2015: Resumen Ejecutivo. London: England; 2015 [consultado 18 de septiembre de 2018]. Disponible en: http://www.helpage.org/silo/files/ndice-global-de-envejecimiento2015-resumen-ejecutivo.pdf

2. Aranco N Stampini M, Ibarrarán P. Medellín N. Panorama de envejecimiento en America Latina y el Caribe [Internet]. Washington: EE. UU.; 2017 [consultado 18 de septiembre de 2018]. Disponible en: https://publications.iadb.org/ bitstream/handle/11319/8757/Panorama-de-envejecimiento-y-dependencia-en-America-Latina-y-el-Caribe.PDF?sequence $=3$

3. Uriarte JD. Resiliencia y envejecimiento. Eur $\mathrm{J}$ investig health psychol educa [Internet]. 2014 [consultado 20 de septiembre de 2018];4(2):67-77. Disponible en: https://formacionasunivep.com/ ejihpe/index.php/journal/article/view/72/59
4. Phillips $S P$, Auais $M$, Belanger $E$, Alvarado $B$, Zunzunegui $M V$. Life-Course social and economic circumstances, gender, and resilience in older adults: The Longitudinal International Mobility in Aging Study (IMIAS). SSM Popul Health [Internet]. 2016 [consultado 20 de septiembre de 2018];2:708-17. Disponible en: https:// www.nchi.nlm.nih.gov/pubmed/29349182

5. Cabanyes J. Resiliencia: una aproximación al concepto. Rev Psiquiatric Salud Ment [Internet]. 2010 [consultado 20 de 
Alejandro Morales-Jinez, Elizabeth Fajardo-Ramos, Francisco Javier López-Rincón, Alicia Ugarte-Esquivel, Yolanda Ivonne Trujillo-León y Luz Elena Rodríguez-Mejía Percepción de las relaciones familiares y nivel de resiliencia en el adulto mayor: un estudio descriptivo-comparativo

septiembre de 2018];3(4):145-51. Disponible en: https://www. researchgate.net/profile/Javier_Cabanyes-Truffino/publication/251719539 Resiliencia_una aproximacion_al_concepto/ links/5a8ff62a45851535bcd46ef1/Resiliencia-una-aproximacion-al-concepto.pdf

6. Garcia-Dia MJ, DiNapoli JM, Garcia-Ona L, Jakubowsky R, O'Flaherty D. Concept Analysis: Resilience. Arch Psychiatr Nurs [Internet]. 2013 [consultado 20 de septiembre de 2018];27(6):26470. Disponible en: https://www.psychiatricnursing.org/article/ S0883-9417(13)00092-7/pdf

7. Arrogante 0. Resiliencia en Enfermería: definición, evidencia empírica e intervenciones. Index Enferm [Internet]. 2015 [consultado 20 de septiembre de 2018];24(4):232-5. Disponible en: http://scielo.isciii.es/scielo.php?script=sci_arttext\&pid=\$1132 $12962015000300009 \&$ lng =es\&nrm=iso\&tlng=es

8. Van Kessel G. The ability of older people to overcome adversity: A review of the resilience concept. Geriatr Nurs [Internet]. 2013 [consultado 20 de septiembre de 2018];34(2):122-7. Disponible en: https://www.ncbi.nlm.nih.gov/pubmed/23332474

9. Von Humboldt $S$, Leal I. Adjustment to aging in late adulthood: A systematic review. Int J Gerontol [Internet]. 2014 [consultado 30 de septiembre de 2018];8(3):108-13. Disponible en: https://www.sciencedirect.com/science/article/pii/ S1873959814000581
10. MacLeod S, Musich S, Hawkins K, Alsgaard K, Wicker ER. The impact of resilience among older adults. Geriatr Nurs [Internet]. 2016 [consultado 30 de septiembre de 2018];37(4):266-72. Disponible en: https://www.ncbi.nlm.nih.gov/pubmed/27055911

11. Schure MB, Odden M, Goins RT. The association of resilience with mental and physical health among older American Indians: The native elder care study. Am Indian Alsk Native Ment Health Res [Internet]. 2013 [consultado 30 de septiembre de 2018];20(2):27-41. Disponible en: https://www.ncbi.nlm.nih.gov/pubmed/23824641

12. Gaffety AE, Bergeman CS, Clark LA, Wirth MM. Aging and the HPA axis: stress and resilience in older adults. Neurosci Biobehav Rev [Internet]. 2016 [consultado 30 de septiembre de 2018];68:928-45 Disponible en: https://www.ncbi.nlm.nih.gov/pubmed/27377692

13. Amar J, Martínez M, Utria L. Nuevo abordaje de la salud considerando la resiliencia. Salud Uninorte Rev [Internet]. 2013 [consultado 30 de septiembre de 2018];29(1):124-33. Disponible en: http:// www.redalyc. org/articulo.oa?id=81728689014

14. Espín AM, Quintero G, Bayarre H. Construcción de un instrumento para medir la percepción de las relaciones familiares del anciano. Estud Interdiscip Envelhec [Internet]. 2001 [consultado 25 de septiembre de 2017];3:55-69. Disponible en: https://seer.ufrgs.br/ RevEnvelhecer/article/view/4669/2587

15. García-León MA, González-Gómez A, Robles-Ortega H, Padilla JL, Peralta-Ramírez MI. Propiedades psicométricas de la Escala de Re- siliencia de Connor y Davidson (CD-RISC) en población española. Anales de psicología [Internet]. 2019 [consultado 14 de enero de 2019];35(1):33-40. Disponible en: https://revistas.um.es/analesps/ article/view/314111/255281

16. Palomar J, Gómez NE. Desarrollo de una escala de medición de la resiliencia con mexicanos (RESI-M). Interdisciplinaria [Internet]. 2010 [consultado 4 de abril de 2019];27(1):7-22. Disponible en: http://www.scielo.org.ar/scielo.php?script=sci_arttext\&pid=\$1668-70272010000100002

17. Quiceno JM, Vinaccia S. Resiliencia y características sociodemográficas en enfermos crónicos. Psicol. caribe [Internet]. 2012 [consultado 4 de abril de 2019];29(1):87-104. Disponible en: https:// www.redalyc.org/pdf/213/21323171006.pdf

18. Morales E, Hernández E, Del Río I, Ortega Y. Caracterización de la percepción de las relaciones familiares de adultos mayores incorporados a círculos de abuelos. Rev haban cien méd [Internet]. 2015 [consultado 4 de abril de 2019];21(2). Disponible en: https://www.medigraphic.com/cgi-bin/new/resumen.cgi?IDARTICULO=61791

19. Abrahante I, Domínguez MC, Hernández LA, Sánchez D. Estado de salud y percepción de las relaciones familiares en el adulto mayor. Rev. cienc. méd. [Internet]. 2016 [consultado 4 de abril de 2019];23(1):28-38. Disponible en: https://www.medigraphic.com/ cgi-bin/new/resumen.cgi?IDARTICULO=68566 